Research Article

\title{
Preliminary BER Study of a TC-OFDM system operating under noisy conditions
}

\author{
S. K. Chronopoulos*, V. Christofilakis, G. Tatsis and P. Kostarakis \\ Physics Department, Electronics-Telecommunications and Applications Laboratory, University of Ioannina, Ioannina, Greece.
}

Received 2 March 2016; Accepted 25 September 2016

\begin{abstract}
Wireless systems contain transmitters and receivers in order to exchange information. In the past decades, this exchange of information was conducted with the use of only one modulated carrier (frequency). As wireless technology evolved, new techniques emerged. One of them was unique for its capability of significant lower data loss compared to others. The main concept of the new technique, commonly known as Orthogonal Frequency Division Multiplexing, was the utilization of various orthogonal carriers (frequencies), which were multiplexed with each other in order to prevent system breakdown in the case of frequency fading. In this paper we simulated our enhanced OFDM platform based on Turbo Coding (TC) in the presence of a noisy channel (AWGN, Phase noise, Rician noise, ITU PA3) exhibiting excellent BER performance.
\end{abstract}

Keywords: OFDM, Turbo codes, Iterative decoding, AWGN, Rician noise, Phase noise, ITU PA3

\section{Introduction}

Noisy environments cause many problems in a system's performance. The first obvious problem is lower data speeds, and one of the most severe problems is frequency fading followed by the total system failure (no data exchange between systems). A smart way of confronting overwhelming noise results is to insert a coding architecture system-in-system under test. One coding technique which is the most promising one, compared to others, is turbo coding (TC). TC is based on a similar transmitting idea like OFDM. Instead of transmitting one stream of codes, the system transmits several streams like OFDM where the system produces various carriers instead of producing only one carrier. In addition to OFDM and turbo coding, an iterative turbo decoder (inside receiver) can literally lift off system's performance. This decoder reevaluates decoded data several times which their number (iterations) are adjusted by the system designer. More iterations conclude to better performance with the drawback of decoding latency. So, the designer must find for every system the best acceptable performance with the fewer possible decoding iterations.

An OFDM system found in many standards [1] is consisted of several stages in order to accomplish the production of various subcarriers (frequencies). Initially, the system drives the information stream through an encoder, then through a modulator and finally is conducted the production of various frequencies (subcarriers) with the help of serial to parallel conversion and IFFT. Also, guard band techniques can be implemented in the system such as zero padding (ZP) and cyclic prefix (CP) [2].

Our platform contained an OFDM transmitter-receiver which utilized both guard band techniques of ZP and CP at the same time. Our unique coding technique [3] were used on the platform as the means to produce Parallel

*E-mail address: schrono@cc.voi.gr

ISSN: $1791-2377$ @ 2016 Eastern Macedonia and Thrace Institute of Technology. All rights reserved. concatenated convolutional codes. At the receiver's part, all the reverse procedures were applied along with our turbo decoding technique [3] being capable of conducting excellent iterative decoding. The channel between transmitter and receiver included AWGN noise, AWGN with phase noise or AWGN with phase noise, Rician fading and ITU PA3 specifications [4]. The idea behind the utilization of these noise types were the fact of taking into consideration the components' imperfections (e.g. amplifier's non-linearity) and the fact that the signal may be consisted of two types of components such as LOS (line of sight) and NLOS (No line of sight).

This paper contains five sections. The second section is the overview of our simulated system along with a brief presentation of our already developed (in simulation level) turbo codes. The third section describes channel's noise characteristics while the fourth section contains simulation settings and results. The fifth section concludes this paper.

\section{Overview of simulated system}

The simulated system (Fig. 1) included a transmitter and a receiver which were connected through a very noisy channel. Both the transmitter's and receiver's architecture were based on the OFDM concept. Multiple orthogonal frequencies were produced with additional guard band techniques such as Zero padding and Cyclic Prefix in order the intersymbol-interference to be eliminated. Then the multiplexed signal passed through the channel with the final destination to be the receiver. At a glance, the OFDM system was designed with the capability to produce thousands of orthogonal frequencies with the minimum interference amongst them [5].

Our already developed coding system [3,6] included the production of parallel turbo codes and a very reliable iterative decoding procedure which were implemented in the transmitter and receiver respectively. Transmitter included the encoder where three convolutional encoders were joined 
in parallel as shown in Fig. 2. Their different encoded streams were concatenated for the production of the final Turbo code. In the receiver the Turbo code was split in its initial coded streams and then these streams where driven to the iterative decoding stage. There, the original data was extracted through an iterative function. This function used for recalculating the final stream by conducting several times the decoding procedure with the use of APP (A Posteriori Probability) decoders and a loop section (Fig. 3).

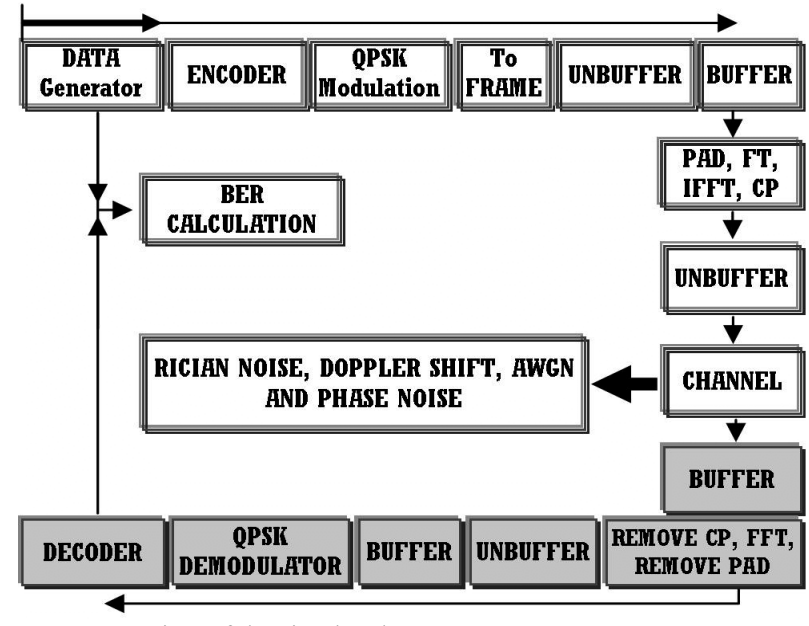

Fig. 1 Overview of the simulated system

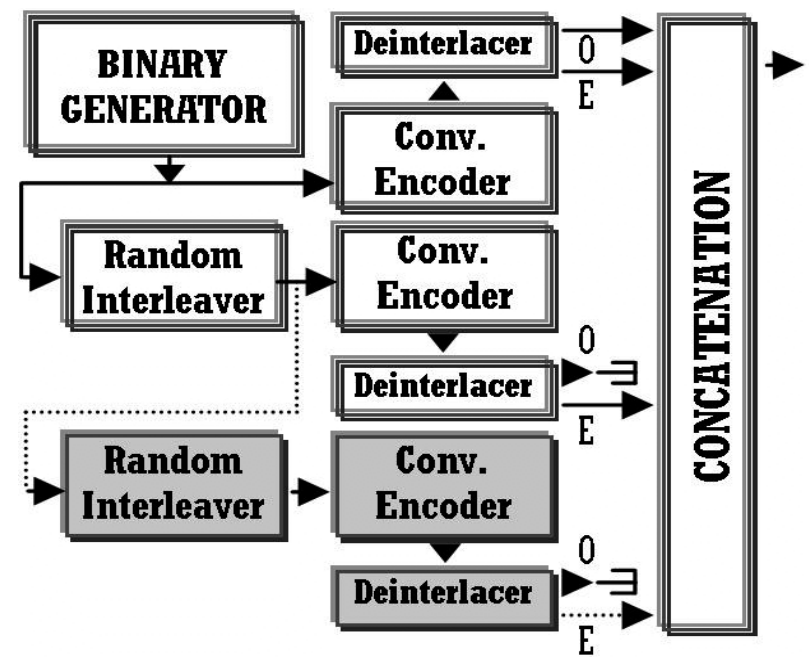

Fig. 2 Turbo encoder

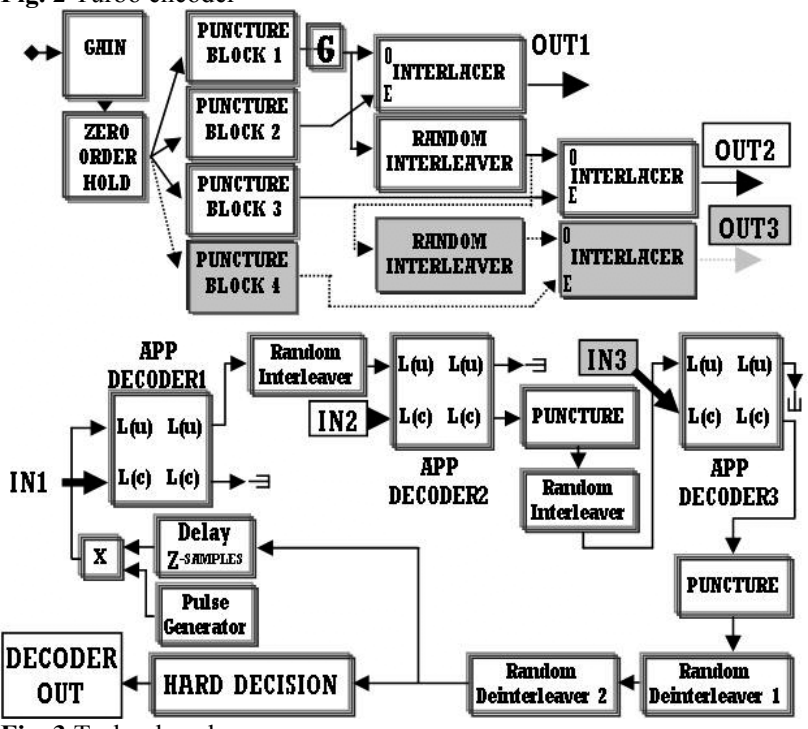

Fig. 3 Turbo decoder

\section{Channel's characteristics}

The channel model in the scenario of Turbo Coded OFDM transmission, included noise types such as Additive White Gaussian Noise (AWGN), Phase Noise, Rician noise and ITU Pedestrian-A.

The AWGN channel referred to a noise which was added in the signal and based in the equation (1). The noise had a flat power spectral density (White noise) and its amplitudes were distributed in Gaussian manner [7].

$y(t)=x(t)+n(t) \quad 0 \leq t \leq T$

Where $y(t)$ is the final signal, $x(t)$ is the transmitted signal without noise, $n(t)$ is the added noise with power spectral density if $\mathrm{N}_{\mathrm{o}} / 2(\mathrm{~W} / \mathrm{Hz})$ and $\mathrm{T}$ is the duration of the transmitted symbol.

Phase noise was included in the simulated Channel's noise diversity for the purpose of including random frequency fluctuations as a means for taking into account hardware impairments (e.g. frequency displacements of carrier's frequency) [8]. Phase noise can be calculated by the equation (2).

$S_{C}(f)=\frac{P_{S}}{P_{S S B}}$

Where $P_{S}$ is the carrier's power and $P_{S S B}$ is the power of a frequency offset with a bandwidth of $1 \mathrm{~Hz}$.

After a signal is transmitted can probably follow different paths towards its destination and can be partially absorbed, scattered, reflected and diffracted depending on the type of channel inside which a system operates. For the purpose of including in our scenario multipatch effects with the line-of-sight (LOS) path to be dominant, Ricean distribution was taken into account for the simulated fading. In this case as it has already mentioned in different words before, the highest percentage of the signal's energy is transmitted through the LOS path. The Ricean-Rice amplitude distribution [9] can be expressed with equation (3).

$$
f_{\text {RICE }}(a)=\left(\begin{array}{r}
\frac{a}{\sigma^{2}} \exp \left(-\frac{a^{2}+\rho^{2}}{2 \sigma^{2}}\right) I_{O}\left(\frac{a \rho}{\sigma^{2}}\right) \text { for } a \geq 0 \\
\text { for } a<0
\end{array}\right)
$$

Where $\rho^{2}$ is the received power of non-fading components, $\mathrm{I}_{\mathrm{O}}$ is the modified Bessel function (of the 1 st kind) and $2 \sigma^{2}$ is the power of non-line-of-sight components.

The inclusion of AWGN, phase noise and Ricean fading in the Channel's impairments give a more realistic working aspect of a system under real conditions. But if a telecommunication system is included in a mobile unit then we must further include in our calculations the times that a channel changes per second. One simple situation could be a pedestrian moving with $3 \mathrm{Km} / \mathrm{h}$ with a maximum Doppler spread (channel alteration) of $5.55 \mathrm{~Hz}$ which declares that the channel changes 5.55 times a second for a working frequency of 2 Ghz. The Tab.1 presents the utilized profile of a Pedestrian's channel (ITU PA3) included in our noise calculations [10].

Table 1. ITU PA3

\begin{tabular}{l|c|c|c|c}
\hline Relative Delay (ns) & 0 & 110 & 190 & 410 \\
\hline Relative Power (dB) & 0.0 & -9.7 & -19.2 & -22.8 \\
\hline
\end{tabular}




\section{Simulations and results}

The simulations (as mentioned before) included four major types of noises for the purpose of investigating the operating capabilities of our TC-OFDM platform [11] which previously had been checked for other impairments such as high PAPR [12,13]. The simulated platform produced 2048 subcarriers in the IFFT output. The coding scheme of the platform included our Turbo Codes [3] with an overall code rate of $1 / 4$. The Turbo codes were consisted of concatenated codes. These codes where produced from convolutional encoders with generator polynomials of 78 and 58 (octal) and with a feedback loop of 7 . The block size was equal to 512 (best performance compared to other block sizes). The decoder section included APP decoders working with logMAP algorithm shown in equation (4). The decoding procedure conducted with a maximum utilized number of three iterations.

$$
V_{M A X} \max ^{*}(X, Y)=\max (X, Y)+\log \left(1+e^{-|Y-X|}\right)
$$

The simulated platform is shown in Fig. 1. The primary data of 1's and 0's was randomly generated with a probability of $50 \%$. The produced stream was encoded, modulated (QPSK), transformed from serial to parallel form along with the appliance of Inverse Fast Fourier Transform (IFFT), and then Zero padding and Cyclic Prefix stages generated the final OFDM stream (which was reinforced with guard band techniques). The OFDM stream passed through the noisy channel and reached the receiver. In the receiver the inverse techniques applied in order to acquire the original coded stream of information which in turn was decoded in the iterative section inside the simulation stage named as DECODER. The calculation of BER was conducted after this stage.

It must be mentioned that a perfect synchronization was assumed between transmitter and receiver by having already implemented all the resultant delays in the calculations. Moreover, perfect angle estimation was conducted relevant to multipath fading. This function was executed by analyzing the complex signal (to amplitude and angle) before entering the channel and in the exit reconstructing the signal by using the original extracted angle information (before the channel). The added phase noise levels were equal to $-50 \mathrm{dBc} / \mathrm{Hz}$ at a frequency offset of $100 \mathrm{~Hz}$. The total added noise quantity (AWGN) to the OFDM signal was calculated as in [11]. The Ricean fading was simulated with $\mathrm{K}$-factors equal to 1 and 2 because they represented the worst case scenario in the literature [14].

The figs 4-7 present the BER performance of the system with or without the addition of Phase Noise. The best results (lowest BER) can be obtained when the system works inside a channel with AWGN and Phase Noise. This is shown clearly in Fig. 7 where all kinds of noise simulations are compared. Notably the system inside an environment with Ricean fading (based on ITU PA3) needs additional $8 \mathrm{~dB}$ in order to obtain the same performance $\left(\mathrm{BER}=10^{-4}\right)$ as when it operates inside an AWGN Channel.

The figs 5-6 show the system's performance inside a channel with Ricean fading $(\mathrm{K}=1$ or $\mathrm{K}=2)$. The system needs at least $6 \mathrm{~dB}$ of signal to noise ratio per bit $\left(\mathrm{E}_{\mathrm{b}} / \mathrm{N}_{\mathrm{o}}\right)$ for exhibiting only 1 received error out of 1000 transmitted bits. The aforementioned performance is very satisfactory in the case of this system which didn't include channel estimation.

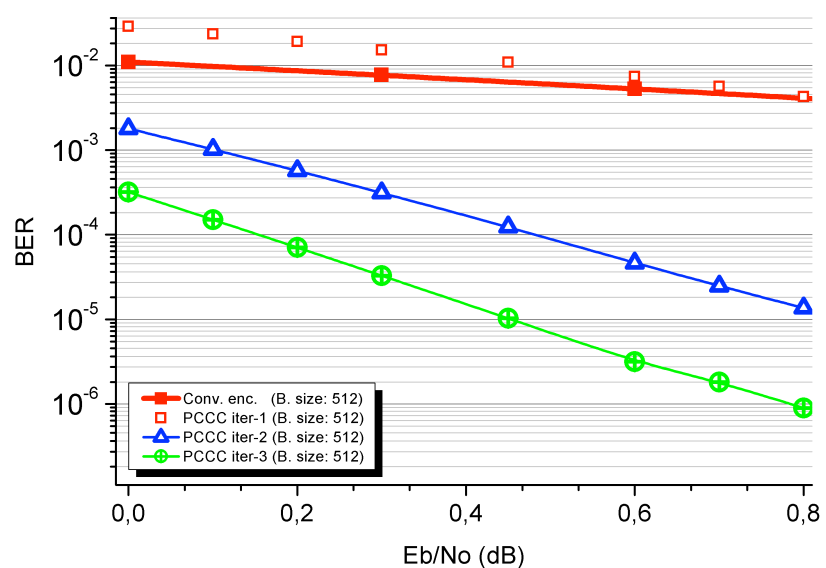

Fig. 4 AWGN with Phase noise

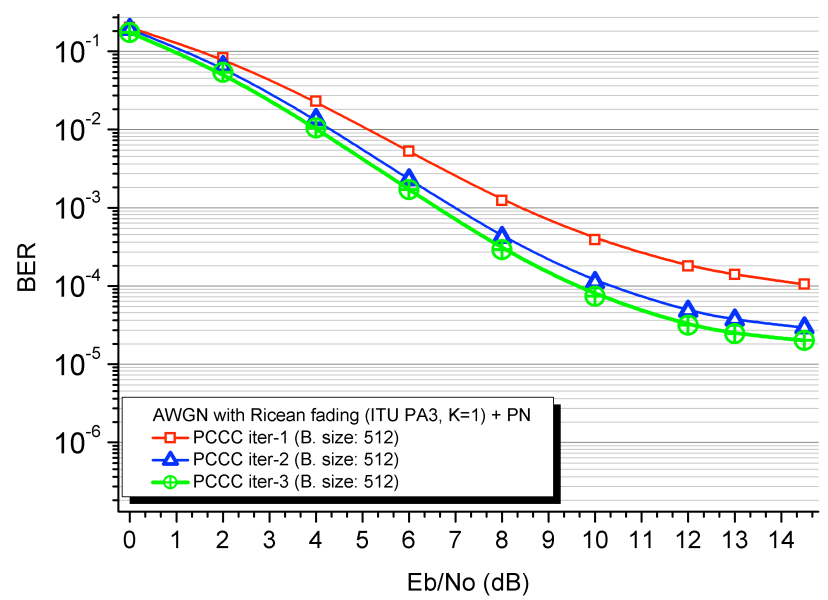

Fig. 5 Rician fading (ITU PA3) with $\mathrm{K}=1$, AWGN and Phase Noise

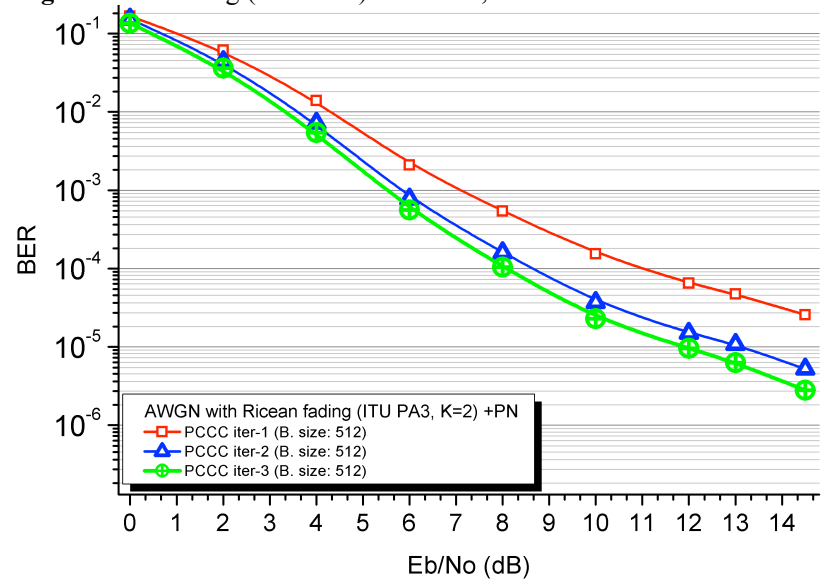

Fig. 6 Rician fading (ITU PA3) with $\mathrm{K}=2$, AWGN and Phase Noise

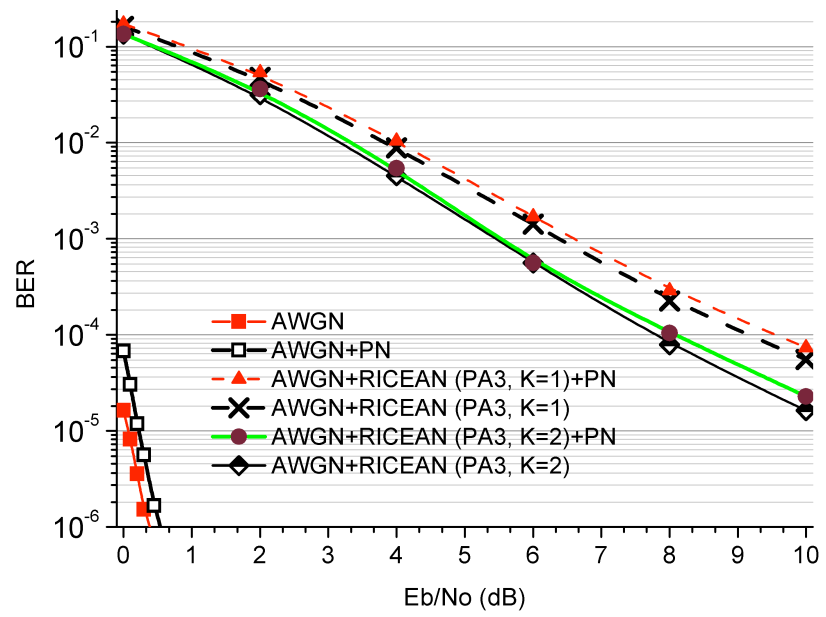

Fig. 7 All kinds of noise simulations 


\section{Conclusions}

Orthogonal frequency division multiplexing technique (OFDM) was combined with Turbo Codes [11] and in this paper our system was simulated with several kinds of noises in order to further investigate the viability of such a scheme. The resulting performance of our system is non-falsifiable as it operates adequately without channel estimation, needing at least $6 \mathrm{~dB}$ of $\mathrm{Eb} / \mathrm{No}$ (for obtaining BER equal to 10-3).

Our TC-OFDM is intended for implementation in a DSP (various stages of this system have already been implemented). Channel estimation will also be investigated for the purpose of implementing the best possible technique in our system.

\section{Acknowledgment}

This research has been co-financed by the European Union (European Social Fund - ESF) and Greek national funds through the Operational Program "Education and Lifelong Learning" of the National Strategic Reference Framework (NSRF) - Research Funding Program: Heracleitus II. Investing in knowledge society through the European Social Fund.

\section{References}

1. W. Henkel, G. Taubock, P. Odling, P. O. Borjesson, N. Petersson and A. Johansson "The Cyclic Prefix of OFDM/DMT-An Analysis," Proceedings of 2002 International Zurich Seminar on Broadband Communications: Access-Transmission-Networking, ETH Zurich, pp. 22-1- 22-3, 2002

2. B. Muquet, Z. Wang, G. B. Giannakis, M. De Courville and P. Duhamel, "Cyclic Prefixing or Zero Padding for Wireless Multicarrier Transmissions?" IEEE Transactions on Communications, Vol. 50, No. 12, pp. 2136-2148, 2002.

3. S. K. Chronopoulos, G. Tatsis and P. Kostarakis, "Turbo Codes-A New PCCC Design," Communications and Network, Vol. 3, No. 4, 2011, pp. 229-234.

4. C. Ye, A. Reznik, G. Sternberg and Y. Shah, "On the Secrecy Capabilities of ITU Channels," In Proceedings of the 66th IEEE Vehicular Technology Conference VTC-2007, IEEE, pp. 2030-203, 2007.

5. S. K. Chronopoulos, C. Votis, V. Raptis, G. Tatsis and P. Kostarakis, "In Depth Analysis of Noise Effects in Orthogonal Frequency Division Multiplexing Systems, Utilising a Large Number of Subcarriers," Proceedings of the 7th International Conference of the Balkan Physical Union, Alexandroupolis, 9-13 September 2009, pp. 967-972. doi:10.1063/1.3322592.

6. S. K Chronopoulos, G. Tatsis, V. Raptis and Panos Kostarakis, “A Parallel turbo encoder-decoder scheme," In 2nd Pan-Hellenic Conference on Electronics and Telecommunications - PACET'12, Organized by the Electronics and the Telecommunications laboratories of the Departments of Physics and Electrical and Computer Engineering, of the Aristotle University of Thessaloniki (AUTh), Greece.

7. J. G. Proakis, "Digital Communications 4th Edition", McGraw-Hill, New York, 2001.

8. Mini-Circuits, "Characterizing and minimizing VCO phase noise," Application Note VCO15-6, 1999. http://www.minicircuits.com/pages/app_notes.html.

9. A. Roca, "Implementation of a WiMAX simulator in Simulink," Diploma Thesis, Engineering Institute-Vienna, 2007.

10. R. Jain, "Channel Models A Tutorial V1.0," CSE574S: Wireless and Mobile Networking, Washington University in St. Louis, 2007.

11. S. K. Chronopoulos, G. Tatsis and P. Kostarakis, "Turbo Coded OFDM with Large Number of Subcarriers," Journal of Signal and Information Processing (JSIP), Vol. 3, No. 2, pp. 161-168, 2012.

12. S. K. Chronopoulos, G. Tatsis, V. Raptis and P. Kostarakis, "Enhanced PAPR in OFDM without Deteriorating BER Performance," International Journal of Communications, Network and System Sciences, Vol. 4, No. 3, pp. 164-169, 2011.

13. S. K. Chronopoulos, V. Christofilakis, G. Tatsis and P. Kostarakis "Reducing Peak-to-Average Power Ratio of a Turbo Coded OFDM," Wireless Engineering and Technology, Vol 3. No.4, pp. 195-202, Oct. 2012.

14. G. G. Messier and J. A. Hartwell "An Empirical Model for Nonstationary Ricean Fading," IEEE Transactions on Vehicular Technology, IEEE, Vol. 58, No. 1, pp. 14-20, 2009. 